\title{
Positive solutions to a class of elastic beam equations with semipositone nonlinearity
}

\author{
by QINGLIU YAO (Nanjing)
}

\begin{abstract}
Let $h \in L^{1}[0,1] \cap C(0,1)$ be nonnegative and $f(t, u, v)+h(t) \geq 0$. We study the existence and multiplicity of positive solutions for the nonlinear fourth-order two-point boundary value problem

$$
u^{(4)}(t)=f\left(t, u(t), u^{\prime}(t)\right), \quad 0<t<1, \quad u(0)=u^{\prime}(0)=u^{\prime}(1)=u^{\prime \prime \prime}(1)=0,
$$

where the nonlinear term $f(t, u, v)$ may be singular at $t=0$ and $t=1$. By constructing a suitable cone and integrating certain height functions of $f(t, u, v)$ on some bounded sets, several new results are obtained. In mechanics, the problem models the deflection of an elastic beam fixed at the left end and clamped at the right end by sliding clamps.
\end{abstract}

1. Introduction. In this paper, we study the existence and multiplicity of positive solutions for the nonlinear fourth-order two-point boundary value problem

$$
(P) \quad\left\{\begin{array}{l}
u^{(4)}(t)=f\left(t, u(t), u^{\prime}(t)\right), \quad 0<t<1, \\
u(0)=u^{\prime}(0)=u^{\prime}(1)=u^{\prime \prime \prime}(1)=0 .
\end{array}\right.
$$

In mechanics, problem $(P)$ models the deflection of an elastic beam fixed at the left end and clamped at the right end by sliding clamps. Here, the function $u^{*} \in C^{1}[0,1]$ is called a positive solution of $(P)$ if $u^{*}$ is a solution of $(P)$ and $u^{*}(t)>0$ for $0<t \leq 1$.

When $f:[0,1] \times(-\infty, \infty) \times(-\infty, \infty) \rightarrow(-\infty, \infty)$ is continuous, the solvability of $(P)$ has been studied by some authors, for example, see [3-5, $10,11]$. As is well known, only positive solutions are significant in most real problems.

If $h(t) \equiv 0$ and $f(t, u, v)$ is nonnegative, the problem $(P)$ is called positive. For the positive problem $(P)$, Dalmasso [3] considered the existence of a single positive solution when $f(t, u, v)=f(u)$ and Yao [10] verified the existence of $n$ positive solutions in some general cases.

2010 Mathematics Subject Classification: 34B15, 34B16, 34B18.

Key words and phrases: nonlinear ordinary differential equation, boundary value problem, positive solution, existence, multiplicity. 
If $h(t) \geq 0$ and $f(t, u, v)+h(t)$ is nonnegative, the problem $(P)$ is called semipositone. To the best of our knowledge, there are no results concerning the solvability of the semipositone problem $(P)$.

In this paper, we will study the existence and multiplicity of positive solutions for the semipositone problem $(P)$ with singularity. The primary motivation comes from the research of other semipositone problems, for example $[1,2,8,12,16]$.

Throughout this paper, we assume

(H1) $h \in L^{1}[0,1] \cap C(0,1)$ is nonnegative and $\bar{M}=\int_{0}^{1} h(t) d t$.

(H2) $f:(0,1) \times[0, \infty) \times[0, \infty) \rightarrow(-\infty, \infty)$ is continuous.

(H3) For each positive number $r>0$, there exists a nonnegative function $j_{r} \in L^{1}[0,1] \cap C(0,1)$ such that

$$
|f(t, u, v)| \leq j_{r}(t), \quad(t, u, v) \in(0,1) \times[0, r] \times[0, r] .
$$

(H4) $f(t, u, v)+h(t) \geq 0$ for $(t, u, v) \in(0,1) \times[0, \infty) \times[0, \infty)$.

The assumptions (H2) and (H3) mean that the nonlinear term $f(t, u, v)$ may be singular at $t=0$ and $t=1$.

The purpose of this paper is to establish several local existence theorems for positive solutions under the assumptions $(\mathrm{H} 1)-(\mathrm{H} 4)$. The proofs are based on the Krasnosel'skil fixed point theorem of cone expansioncompression type. For completeness, we recall it.

Lemma 1.1 (Krasnosel'skiı). Let $X$ be a Banach space, and let $K$ be a cone in $X$. Assume $\Omega_{1}, \Omega_{2}$ are bounded open subsets of $K$ with $0 \in \Omega_{1} \subset$ $\bar{\Omega}_{1} \subset \Omega_{2}$, and let $T: K \rightarrow K$ be a completely continuous operator such that either

(1) $\|T(x)\| \leq\|x\|, x \in \partial \Omega_{1}$ and $\|T(x)\| \geq\|x\|, x \in \partial \Omega_{2}$, or

(2) $\|T(x)\| \geq\|x\|, x \in \partial \Omega_{1}$ and $\|T(x)\| \leq\|x\|, x \in \partial \Omega_{2}$.

Then $T$ has a fixed point in $\bar{\Omega}_{2} \backslash \Omega_{1}$.

In order to apply the fixed point theorem, we need an appropriate cone. By considering the partial derivative $\frac{\partial}{\partial t} G(t, s)$ of the related Green function $G(t, s)$, we obtain a cone which satisfies our requirement.

We will improve the localization method used in [7, 9, 13-15]. The method is applicable only to the boundary value problems with continuous nonlinear term. We will introduce the height functions of the nonlinear term $f(t, u, v)$ on some bounded sets and estimate the growth changes of $f(t, u, v)$ by integrating the height functions. We will apply the improved method to problem $(P)$ and establish the existence of $n$ positive solutions. All results only depend upon the properties of the nonlinear term $f(t, u, v)$ on some bounded sets and are independent of the existence of upper and 
lower solutions. Finally, we will give two examples to illustrate some applications of the main results.

2. Green function and cone. Let $C_{0}^{1}[0,1]=\left\{u \in C^{1}[0,1]: u(0)=0\right\}$ be the Banach space with the norm $\|u\|=\max \left\{\|u\|,\left\|u^{\prime}\right\|\right\}$, where $\|u\|=$ $\max _{0 \leq t \leq 1}|u(t)|$. In addition, let

$$
H(s)=\frac{1}{2} s^{2}, \quad q(t)=t(1-t), \quad p(t)=\int_{0}^{t} q(s) d s=\frac{1}{6} t^{2}(3-2 t) .
$$

Define a cone $K$ of nonnegative functions in $C_{0}^{1}[0,1]$ as follows:

$$
K=\left\{u \in C_{0}^{1}[0,1]: u(t) \geq\|u\| p(t), u^{\prime}(t) \geq\left\|u^{\prime}\right\| q(t), 0 \leq t \leq 1\right\} .
$$

Let $G(t, s)$ be the Green function of the homogeneous linear problem

$$
u^{(4)}(t)=0, \quad 0 \leq t \leq 1, \quad u(0)=u^{\prime}(0)=u^{\prime}(1)=u^{\prime \prime \prime}(1)=0,
$$

that is,

$$
G(t, s)= \begin{cases}\frac{1}{12} s^{2}\left(6 t-2 s-3 t^{2}\right), & 0 \leq s \leq t \leq 1 \\ \frac{1}{12} t^{2}\left(6 s-2 t-3 s^{2}\right), & 0 \leq t \leq s \leq 1\end{cases}
$$

Then

$$
\frac{\partial}{\partial t} G(t, s)= \begin{cases}\frac{1}{2} s^{2}(1-t), & 0 \leq s \leq t \leq 1, \\ \frac{1}{2} t\left(2 s-t-s^{2}\right), & 0 \leq t \leq s \leq 1 .\end{cases}
$$

It is easy to see that $G(t, s) \geq 0$ and $\frac{\partial}{\partial t} G(t, s) \geq 0$ for $0 \leq t, s \leq 1$.

Direct computations give

$$
\max _{0 \leq t, s \leq 1} G(t, s)=G(1,1)=\frac{1}{12}, \quad \max _{0 \leq t, s \leq 1} \frac{\partial}{\partial t} G(t, s)=\frac{\partial}{\partial t} G\left(\frac{2}{3}, \frac{2}{3}\right)=\frac{2}{27} .
$$

If $0<\alpha<\beta \leq 1$, then

$$
\begin{aligned}
\min _{\alpha \leq t, s \leq \beta} G(t, s) & =G(\alpha, \beta)=\frac{\alpha^{2}\left(6 \beta-2 \alpha-3 \beta^{2}\right)}{12}, \\
\min _{\alpha \leq t, s \leq \beta} \frac{\partial}{\partial t} G(t, s) & =\min \left\{\frac{\partial}{\partial t} G(\alpha, \beta), \frac{\partial}{\partial t} G(\beta, \alpha)\right\} \\
& =\min \left\{\frac{\alpha\left(2 \beta-\alpha-\beta^{2}\right)}{2}, \frac{\alpha^{2}(1-\beta)}{2}\right\} .
\end{aligned}
$$

Lemma 2.1. $q(t) H(s) \leq \frac{\partial}{\partial t} G(t, s) \leq H(s)$ for $0 \leq t, s \leq 1$.

Proof. If $0 \leq s \leq t \leq 1$, then

$$
\begin{aligned}
& \frac{\partial}{\partial t} G(t, s)=\frac{1}{2} s^{2}(1-t) \leq \frac{1}{2} s^{2}=H(s), \\
& \frac{\partial}{\partial t} G(t, s)=(1-t) H(s) \geq t(1-t) H(s)=q(t) H(s) .
\end{aligned}
$$


If $0 \leq t \leq s \leq 1$, then

$$
\begin{aligned}
\frac{\partial}{\partial t} G(t, s) & =\frac{1}{2}(1-t) s^{2}-\frac{1}{2}(s-t)^{2} \leq \frac{1}{2}(1-t) s^{2} \leq \frac{1}{2} s^{2}=H(s) \\
\frac{\partial}{\partial t} G(t, s) & =\frac{1}{2}\left(2 t s-t^{2}-t s^{2}\right) \\
& =\frac{1}{2}\left[2 t s-t^{2}-t s^{2}+t(1-t)^{2} s^{2}-t(1-t)^{2} s^{2}\right] \\
& =\frac{1}{2}\left\{t(1-t)^{2} s^{2}+(1-s)[t(s-t)+t s(1-t)]+t^{2}(1-t) s^{2}\right\} \\
& \geq \frac{1}{2} s^{2}\left\{t(1-t)^{2}+t^{2}(1-t)\right\}=q(t) H(s) .
\end{aligned}
$$

Lemma 2.2. For $0 \leq t \leq 1$,

$$
\int_{0}^{1} G(t, s) h(s) d s \leq \frac{2}{9} \bar{M} p(t), \quad \int_{0}^{1} \frac{\partial}{\partial t} G(t, s) h(s) d s \leq \frac{1}{2} \bar{M} q(t) .
$$

Proof. In fact, we have

$$
\begin{gathered}
\frac{G(t, s)}{p(t)}= \begin{cases}\frac{s^{2}\left(6 t-2 s-3 t^{2}\right)}{2 t^{2}(3-2 t)} \leq \frac{\max _{0 \leq t \leq 1}\left(4 t-3 t^{2}\right)}{2 \min _{0 \leq t \leq 1}(3-2 t)}=\frac{2}{9}, & 0 \leq s \leq t \leq 1, \\
\frac{t^{2}\left(6 s-2 t-3 s^{2}\right)}{2 t^{2}(3-2 t)} \leq \frac{\max _{0 \leq t \leq 1}\left(4 t-3 t^{2}\right)}{2 \min _{0 \leq t \leq 1}(3-2 t)}=\frac{2}{9}, & 0 \leq t \leq s \leq 1 ;\end{cases} \\
\frac{\frac{\partial}{\partial t} G(t, s)}{q(t)}= \begin{cases}\frac{s^{2}(1-t)}{2 t(1-t)} \leq \frac{s^{2}}{2 t} \leq \frac{1}{2} t \leq \frac{1}{2}, & 0 \leq s \leq t \leq 1, \\
\frac{t\left(2 s-t-s^{2}\right)}{2 t(1-t)} \leq \frac{s(1-s)}{2(1-t)} \leq \frac{1}{2} s \leq \frac{1}{2}, & 0 \leq t \leq s \leq 1 .\end{cases}
\end{gathered}
$$

It follows that

$$
\begin{aligned}
\frac{\int_{0}^{1} G(t, s) h(s) d s}{p(t)} & \leq \max _{0 \leq t, s \leq 1} \frac{G(t, s)}{p(t)} \int_{0}^{1} h(s) d s \leq \frac{2}{9} \int_{0}^{1} h(s) d s=\frac{2}{9} \bar{M}, \\
\frac{\int_{0}^{1} \frac{\partial}{\partial t} G(t, s) h(s) d s}{q(t)} & \leq \max _{0 \leq t, s \leq 1} \frac{\frac{\partial}{\partial t} G(t, s)}{q(t)} \int_{0}^{1} h(s) d s \leq \frac{1}{2} \int_{0}^{1} h(s) d s=\frac{1}{2} \bar{M} .
\end{aligned}
$$

Lemma 2.3. If $u \in K$ and $\|u\|=r$, then $\left\|u^{\prime}\right\|=r$ and $\frac{1}{6} r \leq\|u\| \leq r$.

Proof. Since $u(0)=0$, we have

$$
u(t)=\int_{0}^{t} u^{\prime}(s) d s \geq\left\|u^{\prime}\right\| \int_{0}^{t} q(s) d s=\left\|u^{\prime}\right\| p(t), \quad 0 \leq t \leq 1 .
$$


It follows that

$$
\frac{1}{6}\left\|u^{\prime}\right\|=\left\|u^{\prime}\right\| \max _{0 \leq t \leq 1} p(t) \leq\|u\| \leq \max _{0 \leq t \leq 1} \int_{0}^{t}\left\|u^{\prime}\right\| d s=\left\|u^{\prime}\right\| .
$$

So, $\left\|u^{\prime}\right\|=r$ and $\frac{1}{6} r \leq\|u\| \leq r$.

3. Related integral operator. Let $u_{0}(t)=\int_{0}^{1} G(t, s) h(s) d s$ for $0 \leq$ $t \leq 1$. Then $u_{0} \in C^{1}[0,1]$ is a nonnegative function such that $u_{0}(0)=$ $u_{0}^{\prime}(0)=u_{0}^{\prime}(1)=u_{0}^{\prime \prime \prime}(1)=0$ and $u_{0}^{(4)}(t)=h(t)$ for $0<t<1$. Moreover, we can prove $u_{0} \in K$ by Lemma 2.1 .

Write $c^{b}=\max \{c, 0\}$.

Consider the fourth-order two-point boundary value problem

$(Q) \quad\left\{\begin{array}{l}u^{(4)}(t)=f\left(t,\left(u(t)-u_{0}(t)\right)^{b},\left(u^{\prime}(t)-u_{0}^{\prime}(t)\right)^{b}\right)+h(t), \quad 0 \leq t \leq 1 \\ u(0)=u^{\prime}(0)=u^{\prime}(1)=u^{\prime \prime \prime}(1)=0\end{array}\right.$

For $u \in K$ and $0 \leq t \leq 1$, define the operator $T$ as follows:

$$
(T u)(t)=\int_{0}^{1} G(t, s)\left[f\left(s,\left(u(s)-u_{0}(s)\right)^{b},\left(u^{\prime}(s)-u_{0}^{\prime}(s)\right)^{b}\right)+h(s)\right] d s .
$$

For convenience, we use the abbreviation

$$
F\left(t,\left(u(t)+u_{0}(t)\right)^{b}\right)=f\left(t,\left(u(t)+u_{0}(t)\right)^{b},\left(u^{\prime}(t)+u_{0}^{\prime}(t)\right)^{b}\right) .
$$

Lemma 3.1. We have $T: K \rightarrow C^{1}[0,1]$ and

$$
(T u)^{\prime}(t)=\int_{0}^{1} \frac{\partial}{\partial t} G(t, s)\left[f\left(s,\left(u(s)-u_{0}(s)\right)^{b},\left(u^{\prime}(s)-u_{0}^{\prime}(s)\right)^{b}\right)+h(s)\right] d s .
$$

Proof. Let $u \in K$. Directly differentiating both sides of the expression of $(T u)(t)$, we get

$$
(T u)^{\prime}(t)=\int_{0}^{1} \frac{\partial}{\partial t} G(t, s)\left[F\left(s,\left(u(s)-u_{0}(s)\right)^{b}\right)+h(s)\right] d s, \quad 0<t<1 .
$$

Since $F\left(\cdot,\left(u(\cdot)-u_{0}(\cdot)\right)^{b}\right) \in C(0,1)$ by the assumption (H2) and

$$
\left|F\left(t,\left(u(t)-u_{0}(t)\right)^{b}\right)\right| \leq j_{\left\|| | u | | \left|+\left\|u_{0} \mid\right\|+1\right.\right.}(t), \quad 0<t<1,
$$

by the assumption (H3), we see that $F\left(\cdot,\left(u(\cdot)-u_{0}(\cdot)\right)^{b}\right)+h(\cdot) \in L^{1}[0,1]$. Therefore, $T,(T(\cdot))^{\prime}: K \rightarrow C[0,1]$ and $T: K \rightarrow C^{1}[0,1]$.

Lemma 3.2. $T: K \rightarrow K$. 
Proof. Let $u \in K$. Since $G(0, s)=0$ for $0 \leq s \leq 1$, we see that $(T u)(0)=0$ from the definition of $T$. By Lemma 3.1,

Hence

$$
(T u)(t)=\int_{0}^{t}(T u)^{\prime}(s) d s=\int_{0}^{t} \int_{0}^{1} \frac{\partial}{\partial s} G(s, \tau)\left[F\left(\tau,\left(u(\tau)-u_{0}(\tau)\right)^{b}\right)+h(\tau)\right] d \tau d s .
$$

$$
\|T u\|=\max _{0 \leq t \leq 1} \int_{0}^{t}(T u)^{\prime}(s) d s \leq \int_{0}^{1}\left\|(T u)^{\prime}\right\| d s=\left\|(T u)^{\prime}\right\| .
$$

On the other hand, by Lemma 2.1,

$$
\begin{aligned}
(T u)(t) & =\int_{0}^{t} \int_{0} \frac{\partial}{\partial s} G(s, \tau)\left[F\left(\tau,\left(u(\tau)-u_{0}(\tau)\right)^{b}\right)+h(\tau)\right] d \tau d s \\
& \geq \int_{0}^{t} q(s) \int_{0}^{1} H(\tau)\left[F\left(\tau,\left(u(\tau)-u_{0}(\tau)\right)^{b}\right)+h(\tau)\right] d \tau d s \\
& \geq \int_{0}^{t} q(s) \max _{0 \leq s \leq 1} \int_{0}^{1} \frac{\partial}{\partial s} G(s, \tau)\left[F\left(\tau,\left(u(\tau)-u_{0}(\tau)\right)^{b}\right)+h(\tau)\right] d \tau d s \\
& =\left\|(T u)^{\prime}\right\| \int_{0}^{t} q(s) d s=\left\|(T u)^{\prime}\right\| p(t) \geq\|T u\| p(t), \\
(T u)^{\prime}(t) & =\int_{0}^{1} \frac{\partial}{\partial t} G(t, s)\left[F\left(s,\left(u(s)-u_{0}(s)\right)^{b}\right)+h(s)\right] d s \\
& \geq q(t) \int_{0}^{1} H(s)\left[F\left(s,\left(u(s)-u_{0}(s)\right)^{b}\right)+h(s)\right] d s \\
& \geq q(t) \max _{0 \leq t \leq 1}^{1} \int_{0}^{1} \frac{\partial}{\partial t} G(t, s)\left[F\left(s,\left(u(s)-u_{0}(s)\right)^{b}\right)+h(s)\right] d s=\left\|(T u)^{\prime}\right\| q(t) .
\end{aligned}
$$

Consequently, $T: K \rightarrow K$.

Lemma 3.3. $T: K \rightarrow K$ is completely continuous.

Proof. By Lemmas 3.1 and 3.2, we have $V \circ J=T$ and $W \circ J=(T(\cdot))^{\prime}$ where

$$
\begin{gathered}
(J u)(t)=F\left(t,\left(u(t)-u_{0}(t)\right)^{b}\right)+h(t), \\
(V u)(t)=\int_{0}^{1} G(t, s) u(s) d s, \quad(W u)(t)=\int_{0}^{1} \frac{\partial}{\partial t} G(t, s) u(s) d s .
\end{gathered}
$$

By the proof of Lemma 3.1, $J: K \rightarrow L^{1}[0,1]$.

Let $u, u_{k} \in K$ and $\left\|\left|u_{k}-u\right|\right\| \rightarrow 0$. Then

$$
\lim _{k \rightarrow \infty} u_{k}(t)=u(t), \quad \lim _{k \rightarrow \infty} u_{k}^{\prime}(t)=u^{\prime}(t), \quad 0 \leq t \leq 1 .
$$


By assumption (H1),

$$
\lim _{k \rightarrow \infty} F\left(t,\left(u_{k}(t)-u_{0}(t)\right)^{b}\right)=F\left(t,\left(u(t)-u_{0}(t)\right)^{b}\right), \quad 0<t<1 .
$$

Since $\left\|u_{k}-u\right\| \rightarrow 0$, there exists $N(u)$ such that, for any $k \geq N(u)$,

$$
0 \leq u_{k}(t) \leq\|u\|+1, \quad 0 \leq u_{k}^{\prime}(t) \leq\|u\|+1, \quad 0 \leq t \leq 1 .
$$

So, for any $k \geq N(u)$,

$$
\left|F\left(t,\left(u_{k}(t)-u_{0}(t)\right)^{b}\right)\right| \leq j_{\|u\|+\left\|u_{0}\right\|+1}(t), \quad 0<t<1 .
$$

Applying the Lebesgue dominated convergence theorem ([6, (12.24), p. 172]), we obtain

$$
\begin{aligned}
\lim _{k \rightarrow \infty} \int_{0}^{1}\left|\left(J u_{k}\right)(t)-(J u)(t)\right| d t & \\
\quad= & \lim _{k \rightarrow \infty} \int_{0}^{1}\left|F\left(t,\left(u_{k}(t)-u_{0}(t)\right)^{b}\right)-F\left(t,\left(u(t)-u_{0}(t)\right)^{b}\right)\right| d t \\
& =\int_{0}^{1} \lim _{k \rightarrow \infty}\left|F\left(t,\left(u_{k}(t)-u_{0}(t)\right)^{b}\right)-F\left(t,\left(u(t)-u_{0}(t)\right)^{b}\right)\right| d t=0 .
\end{aligned}
$$

It follows that $J: K \rightarrow L^{1}[0,1]$ is continuous.

Obviously, $V, W: L^{1}[0,1] \rightarrow C[0,1]$ are bounded linear operators. Thus, $V, W: L^{1}[0,1] \rightarrow C[0,1]$ are continuous.

Applying the Arzelà-Ascoli theorem, we conclude that $V, W: L^{1}[0,1] \rightarrow$ $C[0,1]$ are completely continuous.

Therefore, $T=V \circ J$ and $(T(\cdot))^{\prime}=W \circ J: K \rightarrow C[0,1]$ are completely continuous. It follows that $T: K \rightarrow K$ is completely continuous.

Lemma 3.4. If $\bar{u} \in K$ is a fixed point of the operator $T$ and $\|\bar{u}\|>\frac{4}{3} \bar{M}$, then $u^{*}=\bar{u}-u_{0} \in C^{1}[0,1]$ is a positive solution of problem $(P)$.

Proof. It is easy to prove that fixed points of the operator $T$ are solutions of problem $(Q)$.

Since $\bar{u}$ is a solution of problem $(Q)$, we have

$$
\left\{\begin{array}{l}
\bar{u}^{(4)}(t)=F\left(t,\left(\bar{u}(t)-u_{0}(t)\right)^{b}+h(t), \quad 0<t<1,\right. \\
\bar{u}(0)=\bar{u}^{\prime}(0)=\bar{u}^{\prime}(1)=\bar{u}^{\prime \prime \prime}(1)=0 .
\end{array}\right.
$$

Let $u^{*}(t)=\bar{u}(t)-u_{0}(t), 0 \leq t \leq 1$. Then $u^{*} \in C^{1}[0,1]$. Since $\|\bar{u}\|>\frac{4}{3} \bar{M}$ and Lemma 2.3 , we have $\left\|u^{\prime}\right\|>\frac{4}{3} \bar{M}$ and $\|u\|>\frac{2}{9} \bar{M}$. In addition, by Lemma $2.2, u_{0}(t) \leq \frac{2}{9} \bar{M} p(t)$ and $u_{0}^{\prime}(t) \leq \frac{1}{2} \bar{M} q(t)$ for $0 \leq t \leq 1$. It follows that, for 
$0<t<1$

$$
\begin{gathered}
u^{*}(t)=\bar{u}(t)-u_{0}(t) \geq\|\bar{u}\| p(t)-\frac{2}{9} \bar{M} p(t)>\left(\frac{2}{9} \bar{M}-\frac{2}{9} \bar{M}\right) p(t)=0, \\
\left(u^{*}\right)^{\prime}(t)=\bar{u}^{\prime}(t)-u_{0}^{\prime}(t) \geq\left\|\bar{u}^{\prime}\right\| q(t)-\frac{1}{2} \bar{M} q(t)>\left(\frac{4}{3} \bar{M}-\frac{1}{2} \bar{M}\right) q(t)>0 .
\end{gathered}
$$

From this, $\left(u^{*}(t)\right)^{b}=u^{*}(t)$ and $\left(\left(u^{*}\right)^{\prime}(t)\right)^{b}=\left(u^{*}\right)^{\prime}(t)$ for $0 \leq t \leq 1$. Noticing that $u_{0}^{(4)}(t)=h(t), 0<t<1$ and $u_{0}(0)=u_{0}^{\prime}(0)=u_{0}^{\prime}(1)=u_{0}^{\prime \prime \prime}(1)=0$, we have

$$
\left\{\begin{array}{l}
\left(u^{*}\right)^{(4)}(t)=f\left(t, u^{*}(t),\left(u^{*}\right)^{\prime}(t)\right), \quad 0<t<1, \\
u^{*}(0)=\left(u^{*}\right)^{\prime}(0)=\left(u^{*}\right)^{\prime}(1)=\left(u^{*}\right)^{\prime \prime \prime}(1)=0 .
\end{array}\right.
$$

Since $u^{*}(t) \geq\left(\|\bar{u}\|-\frac{2}{9} \bar{M}\right) p(t)>0,0<t \leq 1, u^{*}$ is a positive solution of problem $(P)$.

4. Main results. We introduce the following height functions and control constants:

$$
\begin{aligned}
& \varphi(t, r)=\max \{f(t, u, v): 0 \leq u \leq r, 0 \leq v \leq r\}+h(t), \\
& \psi(t, r)=\min \left\{f(t, u, v): \begin{array}{l}
\left(\frac{1}{6} r p(t)-\frac{1}{12} \bar{M}\right)^{b} \leq u \leq r, \\
\left(r q(t)-\frac{2}{27} \bar{M}\right)^{b} \leq v \leq r
\end{array}\right\}+h(t), \\
& A=\left[\max _{0 \leq t, s \leq 1} \frac{\partial}{\partial t} G(t, s)\right]^{-1}=\frac{27}{2} \text {, } \\
& B=\left[\min _{\alpha \leq t, s \leq \beta} \frac{\partial}{\partial t} G(t, s)\right]^{-1}=\max \left\{\frac{2}{\alpha\left(2 \beta-\alpha-\beta^{2}\right)}, \frac{2}{\alpha^{2}(1-\beta)}\right\} .
\end{aligned}
$$

By assumptions (H3) and (H4), $\varphi(t, r)$ and $\psi(t, r)$ are integrable and nonnegative. In geometrical terms, $\varphi(t, r)$ is the maximal height function of $f(t, u, v)+h(t)$ on the set $(0,1) \times[0, r] \times[0, r]$, and $\psi(t, r)$ is the minimal height function of $f(t, u, v)+h(t)$ on the set

$$
\left\{(t, u, v): 0 \leq t \leq 1,\left(\frac{1}{6} r p(t)-\frac{1}{12} \bar{M}\right)^{b} \leq u \leq r,\left(r q(t)-\frac{2}{27} \bar{M}\right)^{b} \leq v \leq r\right\} .
$$

We obtain the following local existence theorems.

THEOREM 4.1. Assume that there exist four positive numbers $0<a<b$ and $0<\alpha<\beta<1$ such that $a>\frac{4}{3} \bar{M}$ and one of the following conditions 
is satisfied:

$$
\begin{array}{ll}
\int_{0}^{1} \varphi(t, a) d t \leq A a, & \int_{\alpha}^{\beta} \psi(t, b) d t \geq B b . \\
\int_{\alpha}^{\beta} \psi(t, a) d t \geq B a, & \int_{0}^{1} \varphi(t, b) d t \leq A b .
\end{array}
$$

Then problem $(P)$ has at least one solution $u^{*} \in C^{1}[0,1]$ such that $u^{*}+u_{0}$ $\in K$ and $a \leq\left\|u^{*}+u_{0}\right\| \leq b$.

Proof. Without loss of generality, we only consider case (a1). Define

$$
\Omega_{r}=\{u \in K:\|u\|<r\}, \quad \partial \Omega_{r}=\{u \in K:\|u\|=r\} .
$$

If $u \in \partial \Omega_{a}$, then $\|u\|=a$. By Lemma 2.3, $\left\|u^{\prime}\right\|=a$ and $\|u\| \leq a$. So $0 \leq u(t) \leq a$ and $0 \leq u^{\prime}(t) \leq a$ for $0 \leq t \leq 1$. Thus,

$$
\begin{aligned}
& 0 \leq\left(u(t)-u_{0}(t)\right)^{b} \leq a, \quad 0 \leq\left(u^{\prime}(t)-u_{0}^{\prime}(t)\right)^{b} \leq a, \quad 0 \leq t \leq 1, \\
& f\left(t,\left(u(t)-u_{0}(t)\right)^{b},\left(u^{\prime}(t)-u_{0}^{\prime}(t)\right)^{b}\right)+h(t) \leq \varphi(t, a), \quad 0<t<1 .
\end{aligned}
$$

By Lemma 3.2, Tu $\in$ K. By Lemma 2.3, we have

$$
\begin{aligned}
\|T u\| & =\left\|(T u)^{\prime}\right\| \\
& =\max _{0 \leq t \leq 1} \int_{0}^{1} \frac{\partial}{\partial t} G(t, s)\left[f\left(s,\left(u(s)-u_{0}(s)\right)^{b},\left(u^{\prime}(s)-u_{0}^{\prime}(s)\right)^{b}\right)+h(s)\right] d s \\
& \leq \max _{0 \leq t, s \leq 1} \frac{\partial}{\partial t} G(t, s) \int_{0}^{1}\left[f\left(s,\left(u(s)-u_{0}(s)\right)^{b},\left(u^{\prime}(s)-u_{0}^{\prime}(s)\right)^{b}\right)+h(s)\right] d s \\
& \leq \max _{0 \leq t, s \leq 1} \frac{\partial}{\partial t} G(t, s) \int_{0}^{1} \varphi(s, a) d s \leq A^{-1} A a=a=\|u\| .
\end{aligned}
$$

If $u \in \partial \Omega_{b}$, then $\|u\|=b$. By Lemma $2.3,\left\|u^{\prime}\right\|=b$ and $\frac{1}{6} b \leq\|u\| \leq b$. So

$$
\frac{1}{6} b p(t) \leq u(t) \leq b, \quad b q(t) \leq u^{\prime}(t) \leq b, \quad 0 \leq t \leq 1 .
$$

Simple computations give $\left\|u_{0}\right\| \leq \frac{1}{12} \bar{M}$ and $\left\|u_{0}^{\prime}\right\| \leq \frac{2}{27} \bar{M}$. Therefore,

$$
\begin{gathered}
\left(\frac{1}{6} b p(t)-\frac{1}{12} \bar{M}\right)^{b} \leq\left(u(t)-u_{0}(t)\right)^{b} \leq b, \quad 0 \leq t \leq 1, \\
\left(b q(t)-\frac{2}{27} \bar{M}\right)^{b} \leq\left(u^{\prime}(t)-u_{0}^{\prime}(t)\right)^{b} \leq b, \quad 0 \leq t \leq 1, \\
f\left(t,\left(u(t)-u_{0}(t)\right)^{b},\left(u^{\prime}(t)-u_{0}^{\prime}(t)\right)^{b}\right)+h(t) \geq \psi(t, b), \quad \alpha \leq t \leq \beta .
\end{gathered}
$$

It follows that 


$$
\begin{aligned}
\|T u\| & =\left\|(T u)^{\prime}\right\| \\
& \geq \max _{\alpha \leq t \leq \beta} \int_{\alpha}^{\beta} \frac{\partial}{\partial t} G(t, s)\left[f\left(s,\left(u(s)-u_{0}(s)\right)^{b},\left(u^{\prime}(s)-u_{0}^{\prime}(s)\right)^{b}\right)+h(s)\right] d s \\
& \geq \min _{\alpha \leq t, s \leq \beta} \frac{\partial}{\partial t} G(t, s) \int_{\alpha}^{\beta}\left[f\left(s,\left(u(s)-u_{0}(s)\right)^{b},\left(u^{\prime}(s)-u_{0}^{\prime}(s)\right)^{b}\right)+h(s)\right] d s \\
& \geq \min _{\alpha \leq t, s \leq \beta} \frac{\partial}{\partial t} G(t, s) \int_{\alpha}^{\beta} \psi(s, b) d s \geq B^{-1} B b=b=\|u\| .
\end{aligned}
$$

By Lemmas 3.3 and 1.1, the operator $T$ has a fixed point $\bar{u} \in K$ and $a \leq\|\bar{u}\| \leq b$. Since $a>\frac{4}{3} \bar{M}$, by Lemma 3.4, $u^{*}=\bar{u}-u_{0} \in C^{1}[0,1]$ is a positive solution of problem $(P)$.

Imitating the proof of Theorem 4.1, we can prove the following theorems, where $[c]$ is the integer part of $c$.

THEOREM 4.2. Assume that there exist five positive numbers $0<a<$ $b<c$ and $0<\alpha<\beta<1$ such that $a>\frac{4}{3} \bar{M}$ and one of the following conditions is satisfied:

$$
\begin{array}{lll}
\int_{0}^{1} \varphi(t, a) d t \leq A a, & \int_{\alpha}^{\beta} \psi(t, b) d t>B b, & \int_{0}^{1} \varphi(t, c) d t \leq A c ; \\
\int_{\alpha}^{\beta} \psi(t, a) d t \geq B a, & \int_{0}^{1} \varphi(t, b) d t<A b, & \int_{\alpha}^{\beta} \psi(t, c) d t \geq B c .
\end{array}
$$

Then problem $(P)$ has at least two positive solutions $u_{1}^{*}, u_{2}^{*} \in C^{1}[0,1]$ such that $u_{1}^{*}+u_{0}, u_{2}^{*}+u_{0} \in K$ and $a \leq\left\|u_{1}^{*}+u_{0}\right\|<b<\left\|u_{2}^{*}+u_{0}\right\| \leq c$.

THEOREM 4.3. Assume that there exist six positive numbers $0<a<$ $b<c<d$ and $0<\alpha<\beta<1$ such that $a>\frac{4}{3} \bar{M}$ and one of the following conditions is satisfied:

$$
\begin{array}{ll}
\int_{0}^{1} \varphi(t, a) d t \leq A a, & \int_{\alpha}^{\beta} \psi(t, b) d t>B b \\
\int_{0}^{1} \varphi(t, c) d t<A c, & \int_{\alpha}^{\beta} \psi(t, d) d t \geq B d ; \\
\int_{\alpha}^{\beta} \psi(t, a) d t \geq B a, & \int_{0}^{1} \varphi(t, b) d t<A b, \\
\int_{\alpha} \psi(t, c) d t>B c, & \int_{0}^{1} \varphi(t, d) d t \leq A d .
\end{array}
$$


Then problem $(P)$ has at least three positive solutions $u_{1}^{*}, u_{2}^{*}, u_{3}^{*} \in C^{1}[0,1]$ such that $u_{i}^{*}+u_{0} \in K, i=1,2,3$, and

$$
a \leq\left\|u_{1}^{*}+u_{0}\right\|<b<\left\|u_{2}^{*}+u_{0}\right\|<c<\left\|u_{3}^{*}+u_{0}\right\| \leq d .
$$

TheOREM 4.4. Assume that there exist $n+3$ positive numbers $0<a_{1}<$ $a_{2}<\cdots<a_{n+1}$ and $0<\alpha<\beta<1$ such that $a_{1}>\frac{4}{3} \bar{M}$ and one of the following conditions is satisfied:

$$
\begin{aligned}
& \int_{0}^{1} \varphi\left(t, a_{2 k-1}\right) d t<A a_{2 k-1}, \quad k=1, \ldots,[(n+2) / 2], \\
& \int_{\alpha}^{\beta} \psi\left(t, a_{2 k}\right) d t>B a_{2 k}, \quad k=1, \ldots,[(n+1) / 2] ; \\
& \int_{\alpha}^{\beta} \psi\left(t, a_{2 k-1}\right) d t>B a_{2 k-1}, \quad k=1, \ldots,[(n+2) / 2],
\end{aligned}
$$

$$
\int_{0}^{1} \varphi\left(t, a_{2 k}\right) d t<A a_{2 k}, \quad k=1, \ldots,[(n+1) / 2] .
$$

Then problem $(P)$ has at least $n$ solutions $u_{k}^{*} \in C^{1}[0,1], k=1, \ldots, n$, such that $u_{k}^{*}+u_{0} \in K$ and $a_{k}<\left\|u_{k}^{*}+u_{0}\right\|<a_{k+1}$.

Theorem 4.5 gives a sufficient condition for the existence of infinitely many positive solutions.

TheOREM 4.5. Assume that there exist two positive numbers $0<\alpha<$ $\beta<1$ such that

$$
\liminf _{r \rightarrow \infty} \frac{1}{r} \int_{0}^{1} \varphi(t, r) d t<A, \quad \limsup _{r \rightarrow \infty} \frac{1}{r} \int_{\alpha}^{\beta} \psi(t, r) d t>B .
$$

Then problem $(P)$ has a sequence $\left\{u_{k}^{*}\right\}_{k=1}^{\infty} \subset C^{1}[0,1]$ of positive solutions such that $u_{k}^{*}+u_{0} \in K$ and $\left\|u_{k}^{*}\right\| \rightarrow \infty$.

Proof. The assumption implies that there exists a sequence $\left\{r_{k}\right\}_{k=1}^{\infty}$ of positive numbers such that

$$
\begin{aligned}
\frac{4}{3} \bar{M} \leq r_{1}<r_{2}<\cdots & \text { and } \quad r_{k} \rightarrow \infty \\
\int_{0}^{1} \varphi\left(t, r_{2 k-1}\right) d t \leq A r_{2 k-1}, & \int_{\alpha}^{\beta} \psi\left(t, r_{2 k}\right) d t \geq B r_{2 k} .
\end{aligned}
$$

By Theorem 4.1, for every $k$, there exists a positive solution $u_{k}^{*} \in C^{1}[0,1]$ such that $u_{k}^{*}+u_{0} \in K$ and $r_{2 k-1} \leq\left\|u_{k}^{*}+u_{0}\right\| \leq r_{2 k}$. Consequently, we have $\left\|u_{k}^{*}\right\| \rightarrow \infty$. 


\section{Remarks and examples}

Remark 5.1. We consider the special case of $f(t, u, v)=f(t, u)$. Let $C[0,1]$ be the Banach space with $\|u\|=\max _{0 \leq t \leq 1}|u(t)|$ and

$$
\tilde{K}=\{u \in C[0,1]: u(0)=0, u(t) \geq\|u\| p(t), 0 \leq t \leq 1\} .
$$

In this remark, we assume that

$(\mathrm{H} 1)^{\prime} h \in L^{1}[0,1] \cap C(0,1)$ is nonnegative and $\bar{M}=\int_{0}^{1} h(t) d t$.

$(\mathrm{H} 2)^{\prime} f:(0,1) \times[0, \infty) \rightarrow(-\infty, \infty)$ is continuous.

(H3) ${ }^{\prime}$ For each positive number $r>0$, there exists a nonnegative function $j_{r} \in L^{1}[0,1] \cap C(0,1)$ such that $|f(t, u)| \leq j_{r}(t)$ for $(t, u) \in$ $(0,1) \times[0, r]$.

$(\mathrm{H} 4)^{\prime} f(t, u)+h(t) \geq 0,(t, u) \in(0,1) \times[0, \infty)$.

Consider the fourth-order boundary value problem

$$
(\tilde{P}) \quad\left\{\begin{array}{l}
u^{(4)}(t)=f(t, u(t)), \quad 0<t<1, \\
u(0)=u^{\prime}(0)=u^{\prime}(1)=u^{\prime \prime \prime}(1)=0 .
\end{array}\right.
$$

We will use the following height functions and control constants:

$$
\begin{aligned}
\tilde{\varphi}(t, r) & =\max \{f(t, u): 0 \leq u \leq r\}+h(t), \\
\tilde{\psi}(t, r) & =\min \left\{f(t, u):\left(r p(t)-\frac{1}{12} \bar{M}\right)^{b} \leq u \leq r\right\}+h(t), \\
\tilde{A} & =\left[\max _{0 \leq t, s \leq 1} G(t, s)\right]^{-1}=12, \\
\tilde{B} & =\left[\min _{\alpha \leq t, s \leq \beta} G(t, s)\right]^{-1}=\frac{12}{\alpha^{2}\left(6 \beta-2 \alpha-3 \beta^{2}\right)} .
\end{aligned}
$$

Imitating Theorem 4.1, we can prove the following local existence theorem.

Existence TheORem. Assume that there exist four positive numbers $0<a<b$ and $0<\alpha<\beta \leq 1$ such that $a>\frac{2}{9} \bar{M}$ and one of the following conditions is satisfied:

$$
\begin{aligned}
& \int_{0}^{1} \tilde{\varphi}(t, a) d t \leq \tilde{A} a \quad \text { and } \quad \int_{\alpha}^{\beta} \tilde{\psi}(t, b) d t \geq \tilde{B} b \\
& \int_{\alpha}^{\beta} \tilde{\psi}(t, a) d t \geq \tilde{B} a \quad \text { and } \quad \int_{0}^{1} \tilde{\varphi}(t, b) d t \leq \tilde{A} b .
\end{aligned}
$$

Then problem $(\tilde{P})$ has at least one solution $u^{*} \in C[0,1]$ such that $u^{*}+u_{0} \in \tilde{K}$ and $a \leq\left\|u^{*}+u_{0}\right\| \leq b$.

By applying the existence theorem and changing corresponding constants, we can obtain results similar to Theorems 4.2-4.5. 
REMARK 5.2. In Theorems 4.1-4.4, one of the crucial conditions is the inequality $\int_{\alpha}^{\beta} \psi(t, r) d t \geq B r$. Hence, we need to choose the subinterval $[\alpha, \beta]$. The choice depends upon the behavior of the nonlinear term $f(t, u, v)$ or $f(t, u)$.

In the studies of other nonlinear boundary value problems, the authors usually choose $\alpha=1 / 4, \beta=3 / 4$. The following example shows that the choice is not unique and there may be a finer choice.

Consider the nonlinear boundary value problem

$$
\left\{\begin{array}{l}
u^{(4)}(t)=240 \sqrt{\max \{0, t-3 / 4\} u(t)}, \quad 0 \leq t \leq 1, \\
u(0)=u^{\prime}(0)=u^{\prime}(1)=u^{\prime \prime \prime}(1)=0 .
\end{array}\right.
$$

In this problem, $f(t, u)=240 \sqrt{\max \{0, t-3 / 4\}}$. Hence $f:[0,1] \times[0, \infty) \rightarrow$ $[0, \infty)$ is continuous, $h(t) \equiv 0$ and $\bar{M}=0$.

If $\alpha=1 / 4, \beta=3 / 4$, then $\tilde{\psi}(t, r) \equiv 0$ for any $r>0$. Hence, we cannot derive the existence of any positive solution from Remark 5.1.

However, the problem has a positive solution for other $[\alpha, \beta]$. In fact, let $\alpha=13 / 16, \beta=1$; then $\tilde{A}=12, \tilde{B}=\frac{24576}{1859} \approx 13.2200$. Obviously,

$$
\begin{gathered}
\tilde{\varphi}(t, 100) \leq 240 \max \left\{\frac{1}{2} \sqrt{u}: 0 \leq u \leq 100\right\}=1200, \quad 0 \leq t \leq 1, \\
\int_{0}^{1} \tilde{\varphi}(t, 100) d t=1200=100 \tilde{A} .
\end{gathered}
$$

Since $\min _{13 / 16 \leq t \leq 1} p(t)=\frac{1859}{12288}$, we have, for $13 / 16 \leq t \leq 1$,

$$
\begin{aligned}
\tilde{\psi}\left(t, \frac{1}{10}\right) & \geq 240 \min \left\{\frac{1}{4} \sqrt{u}: \frac{1}{10} p(t) \leq u \leq \frac{1}{10}\right\}, \\
& \geq 240 \min \left\{\frac{1}{4} \sqrt{u}: \frac{1859}{10 \times 12288} \leq u \leq \frac{1}{10}\right\} \\
& =60 \sqrt{\frac{1859}{10 \times 12288}} \approx 7.3799 .
\end{aligned}
$$

Thus $\int_{13 / 16}^{1} \tilde{\psi}(t, 1 / 10) d t \geq 1.3837>1.3220 \approx \frac{1}{10} \tilde{B}$. By Remark 5.1, the problem has a positive solution $u^{*} \in C[0,1]$ and $1 / 10 \leq\left\|u^{*}\right\| \leq 100$.

In Examples 5.3 and 5.4, we let $\alpha=1 / 4, \beta=3 / 4$. So, $A=27 / 2, B=128$.

EXAmple 5.3. Consider the semipositone boundary value problem

$$
\left\{\begin{array}{l}
u^{(4)}(t)=\frac{1}{2}\left[\left(u^{\prime \prime}\right)^{2}(t)+u^{2}(t)\right]-1 / \sqrt{\min \{t, 1-t\}}, \quad 0<t<1, \\
u(0)=u^{\prime}(0)=u^{\prime}(1)=u^{\prime \prime \prime}(1)=0 .
\end{array}\right.
$$

In the problem, the nonlinear term $f(t, u)=\frac{1}{2}\left[u^{2}+v^{2}\right]-1 / \sqrt{\min \{t, 1-t\}}$ is singular at $t=0, t=1$ and has no lower bound for any fixed $(u, v)$. 
Let $h(t)=1 / \sqrt{\min \{t, 1-t\}}$. Then the assumptions (H1)-(H4) hold and $\bar{M}=2 \sqrt{2}$.

It is easy to see that

$$
\begin{gathered}
\varphi(t, 10)=\max \left\{\frac{1}{2}\left[u^{2}+v^{2}\right]: 0 \leq u, v \leq 10\right\}=100, \quad 0 \leq t \leq 1, \\
\int_{0}^{1} \varphi(t, 10) d t=100<135=10 \mathrm{~A} .
\end{gathered}
$$

Since $\min _{1 / 4 \leq t \leq 3 / 4} p(t)=5 / 192, \min _{1 / 4 \leq t \leq 3 / 4} q(t)=1 / 4$, we see that if $r>64 \sqrt{2} \approx 90.5097$, then $\frac{1}{6} r p(t)-\frac{1}{12} \bar{M}>0$ and $r q(t)-\frac{2}{27} \bar{M}>0$ for $1 / 4 \leq t \leq 3 / 4$. It follows that, for $1 / 4 \leq t \leq 3 / 4$,

$$
\begin{aligned}
\psi(t, 9000) & =\min \left\{\frac{1}{2}\left[u^{2}+v^{2}\right]: \begin{array}{c}
1500 p(t)-\frac{1}{12} \bar{M} \leq u \leq 9000, \\
9000 q(t)-\frac{2}{27} \bar{M} \leq v \leq 9000
\end{array}\right\} \\
& \geq \min \left\{\frac{1}{2}\left[u^{2}+v^{2}\right]: \begin{array}{c}
\frac{7500}{192}-\frac{\sqrt{2}}{6} \leq u \leq 9000, \\
\frac{9000}{4}-\frac{4 \sqrt{2}}{27} \leq v \leq 9000
\end{array}\right\} \\
& =\frac{1}{2}\left\{\left[\frac{625}{16}-\frac{\sqrt{2}}{6}\right]^{2}+\left[2250-\frac{4 \sqrt{2}}{27}\right]^{2}\right\} \approx 2531532.3 .
\end{aligned}
$$

Hence, $\int_{1 / 4}^{3 / 4} \psi(t, 9000) d t \geq 1265766.1>1152000=9000 B$.

Since $10>8 \sqrt{2} / 3=\frac{4}{3} \bar{M}$, by Theorem 4.1 the problem has a positive solution $u^{*} \in C^{1}[0,1]$ such that $u^{*}+u_{0} \in K$ and $10 \leq\left\|u^{*}+u_{0}\right\| \leq 9000$. Here, $u_{0}(t)=\int_{0}^{1} G(t, s) d s / \sqrt{\min \{s, 1-s\}}$.

EXAMPLE 5.4. Let $Q_{1}=12, X_{1}=4 Q_{1}+32, Y_{1}=\frac{21 B}{9 A} X_{1}+8$ and $Q_{n+1}=$ $Y_{n}+8, X_{n+1}=4 Q_{n+1}+32, Y_{n+1}=\frac{21 B}{9 A} X_{n+1}+8, n=1,2, \ldots$ In addition, let $Z_{n}=\frac{21}{10} B X_{n}, n=1,2, \ldots$

Let $g(u)$ be the function whose graph is an infinite broken line $(0,0) \rightarrow$ $(10,0) \rightarrow\left(Q_{1}, Z_{1}\right) \rightarrow\left(Y_{1}, Z_{1}\right) \rightarrow\left(Q_{2}, Z_{2}\right) \rightarrow\left(Y_{2}, Z_{2}\right) \rightarrow\left(Q_{3}, Z_{3}\right) \rightarrow$ $\left(Y_{3}, Z_{3}\right) \rightarrow \cdots$.

Consider the semipositone boundary value problem

$$
\left\{\begin{array}{l}
u^{(4)}(t)=g\left(u^{\prime}(t)\right)+\sin ^{2}(u(t))-27 \sqrt{2} / \sqrt{\min \{t, 1-t\}}, \quad 0<t<1, \\
u(0)=u^{\prime}(0)=u^{\prime}(1)=u^{\prime \prime \prime}(1)=0 .
\end{array}\right.
$$

Here $f(t, u, v)=g(v)+\sin ^{2} u-27 \sqrt{2} / \sqrt{\min \{t, 1-t\}}$ is singular at $t=0, t=1$ and $g(v)$ is continuous and nondecreasing. Let $h(t)=27 \sqrt{2} / \sqrt{\min \{t, 1-t\}}$. Then the assumptions (H1)-(H4) hold and $\bar{M}=108$. In addition, $\min _{1 / 4 \leq t \leq 3 / 4} q(t)=1 / 4$. 
It is easy to see that, for $n=1,2, \ldots$ and $0 \leq t \leq 1$,

$$
\begin{aligned}
\varphi\left(t, Y_{n}\right) & \leq \max \left\{g(v)+1: 0 \leq v \leq Y_{n}\right\}=Z_{n}+1=\frac{21}{10} B X_{n}+1 \\
& =\frac{21}{10} B \cdot \frac{9 A}{21 B}\left(Y_{n}-8\right)+1=\frac{9}{10} A Y_{n}-\frac{72}{10} A+1 .
\end{aligned}
$$

It follows that

$$
\int_{0}^{1} \psi\left(t, Y_{n}\right) d t \leq \frac{9}{10} A Y_{n}+1 \quad \text { and } \quad \liminf _{r \rightarrow \infty} \frac{1}{r} \int_{0}^{1} \varphi(t, r) d t<A .
$$

On the other hand, for $1 / 4 \leq t \leq 3 / 4$ and $n=1,2, \ldots$,

$$
\begin{aligned}
\psi\left(t, X_{n}\right) & \geq \min \left\{g(v): X_{n} q(t)-\frac{2}{27} \bar{M} \leq v \leq X_{n}\right\} \\
& \geq \min \left\{g(v): \frac{1}{4} X_{n}-8 \leq v \leq X_{n}\right\} \\
& =\min \left\{g(v): Q_{n} \leq v \leq X_{n}\right\}=Z_{n}=\frac{21}{10} B X_{n} .
\end{aligned}
$$

Thus,

$$
\int_{1 / 4}^{3 / 4} \psi\left(t, X_{n}\right) d t \geq \frac{21}{20} B X_{n} \text { and } \quad \limsup _{r \rightarrow \infty} \frac{1}{r} \int_{1 / 4}^{3 / 4} \psi(t, r) d t>B .
$$

By Theorem 4.5, the problem has a sequence $\left\{u_{k}^{*}\right\}_{k=1}^{\infty} \subset C^{1}[0,1]$ of positive solutions such that $\left\|u_{k}^{*}\right\| \rightarrow \infty$.

Examples 5.3 and 5.4 illustrate that our method can deal with more complicated problems.

\section{References}

[1] R. P. Agarwal and D. O'Regan, A note on existence of nonnegative solutions to singular semipositone problems, Nonlinear Anal. 36 (1999), 615-622.

[2] V. Anuladha, D. D. Hai and R. Shivaji, Existence results for superlinear semipositone boundary value problems, Proc. Amer. Math. Soc. 124 (1996), 757-763.

[3] R. Dalmasso, Uniqueness of positive solutions for some nonlinear fourth order equations, J. Math. Anal. Appl. 201 (1996), 152-168.

[4] M. B. M. Elgindi and Z. Guan, On the global solvability of a class of fourth-order nonlinear boundary value problems, Int. J. Math. Sci. 20 (1997), 257-262.

[5] C. P. Gupta, Existence and uniqueness theorems for the bending of an elastic beam equation, Appl. Anal. 26 (1988), 289-304.

[6] E. Hewitt and K. Stromberg, Real and Abstract Analysis, Springer, Berlin, 1975.

[7] K. Q. Lan, Multiple positive solutions of semilinear differential equations with singularities, J. London Math. Soc. 63 (2001), 690-704.

[8] S. Stanek, Positive solutions for a class of singular semipositone boundary value problems, Math. Comput. Model. 33 (2001), 353-361. 
[9] P. J. Y. Wong and R. P. Agarwal, Multiple solutions for a system of $\left(n_{i}, p_{i}\right)$ boundary value problems, Z. Anal. Anwendungen 19 (2000), 511-528.

[10] Q. Yao, Positive solutions of nonlinear elastic beam equations with a fixed end and a movable end, J. Harbin Inst. Technol. 13 (2006), 545-548.

[11] —, An existence theorem of nonlinear elastic beam equations fixed at left and clamped at right by sliding clamps, Acta Anal. Funct. Appl. 9 (2007), 112-116.

[12] - Existence of $n$ solutions and/or positive solutions to a semipositone elastic beam equation, Nonlinear Anal. 66 (2007), 138-150.

[13] - On the positive solutions of Lidstone boundary value problems, Appl. Math. Comput. 137 (2003), 477-485.

[14] -, Existence, multiplicity and infinite solvability of positive solutions for one-dimensional p-Laplacian, Acta Mat. Sinica (English Ser.) 21 (2005), 691-698.

[15] -, Positive solutions of nonlinear elastic beam equation rigidly fastened on the left and simply supported on the right, Nonlinear Anal. 69 (2008), 1570-1580.

[16] X. Zhang and L. Liu, Positive solutions of superlinear semipositone singular Dirichlet boundary value problems, J. Math. Anal. Appl. 315 (2006), 525-537.

Qingliu Yao

Department of Applied Mathematics

Nanjing University of Finance and Economics

Nanjing 210003, P.R. China

E-mail: yaoqingliu2002@hotmail.com

Received 27.11.2008

and in final form 7.6.2009 